\title{
Output Feedback Control of a Ball and Beam System Based on Jacobian Linearization under Sensor Noise

\author{
Hyun-Do Kim ${ }^{1, a}$, Kyung-Hyun Oh ${ }^{1, b}$ and Ho-Lim Choi ${ }^{1, c}$
}

${ }^{1}$ Dept. of Electrical Engineering, Dong-A University, 840 Hadan2-dong, Saha-gu, Busan, Korea

ahyundo10@naver.com, 'byuen001@naver.com, 'chlchoi@dau.ac.kr (corresponding author)

Keywords: a ball and beam system, Jacobian linearization, sensor noise, output feedback

Abstract. In this paper, we consider a control problem of a ball and beam system with sensor noise on feedback sensor. If sensor noise exists in sensor's signal, it can make feedback signals deformed and then it can lead to control performance degradation or even system failure. Therefore, we need to design a robust controller to deal with the possible sensor noise in the feedback information. We develop an output feedback controller with a gain-scaling factor in order to minimize the effect of AC sensor noise in output feedback information. Our proposed controller is applied to a ball and beam system and verified by analysis and simulation. As a result, our controller reduces the effect of sensor noise to arbitrarily small by increasing a gain-scaling factor.

\section{Introduction}

We consider a control problem of a ball and beam system with sensor noise on feedback channel. Control systems operate via measurement data and it is usually assumed that the measured signals are clean. If sensor noise exists in sensor's signal, it can make feedback signals deformed and then it can result in performance degradation or even system failure. Therefore, we need to design a robust controller to accommodate the possible sensor noise in the feedback information [2],[5],[6].

In this paper, we assume that an output sensor of a ball and beam system is coupled with AC noise signal. We apply Jacobian linearization to a ball and beam system and propose an output feedback controller compensating sensor noise of feedback sensor for a ball and beam system. Our controller is equipped with a gain-scaling factor to minimize the effect of sensor noise in output feedback information. We give a theoretical analysis of the controlled system using Lyapunov stability theorems and Laplace transform [2],[4]. We illustrate the improved control performance via simulation for a ball and beam system.

\section{A ball and beam system model based on Jacobian linearization and sensor noise}

The ball and beam system can be modeled as [7]

$$
\begin{aligned}
& \ddot{r}=K_{b b} \sin \theta \\
& \theta=-\frac{1}{\mu} \dot{\theta}+\frac{K_{s r v}}{\mu} u
\end{aligned}
$$

where $r$ is the position of ball, $\theta$ is the angle of motor, $K_{b b}$ is the model gain of the ball and beam system, $K_{s r v}$ is a steady-state gain, $\mu$ is a time constant and $u$ is an input motor voltage.

We define the states of the system as follows

$$
\begin{aligned}
& r=x_{1}, \dot{r}=\dot{x}_{1}=x_{2}, \ddot{r}=\dot{x}_{2} \\
& \theta=x_{3}, \dot{\theta}=\dot{x}_{3}=x_{4}, \ddot{\theta}=\dot{x}_{4}
\end{aligned}
$$

Then, we obtain the following state equation

$$
\dot{x}_{1}=x_{2}, \dot{x}_{2}=K_{b b} \sin x_{3}, \dot{x}_{3}=x_{4}, \dot{x}_{4}=-\frac{1}{\mu} x_{4}+\frac{K_{s r v}}{\mu} u, y=x_{1}
$$


The Jacobian linearlized system is as follows

$$
\begin{aligned}
& \dot{x}=A x+B v+\delta(x) \\
& y=C x
\end{aligned}
$$

where $x=\left[x_{1}, x_{2}, x_{3}, x_{4}\right]^{T}$ is the state, $v=\frac{K_{s v}}{\mu} u$ is an internal input, $u \in R$ is an input, $y \in R$ is an output, $\delta(x)=\left[0,0,0, \delta_{4}(x)\right]^{T}$ and $\delta_{4}(x)=-\frac{1}{\mu} x_{4}$. The system matrices are

$$
A=\left[\begin{array}{cccc}
0 & 1 & 0 & 0 \\
0 & 0 & K_{b b} & 0 \\
0 & 0 & 0 & 1 \\
0 & 0 & 0 & 0
\end{array}\right], \quad B=\left[\begin{array}{l}
0 \\
0 \\
0 \\
1
\end{array}\right], \quad C=\left[\begin{array}{llll}
1 & 0 & 0 & 0
\end{array}\right]
$$

We suppose that sensor noise enters at the output sensor of a ball and beam system and the measured output becomes as follows

$$
\bar{y}=y+s(t)
$$

where $s(t)$ is sensor noise. Sensor noise is often modeled as a sinusoidal function [1]. Therefore, we introduce the following condition on $s(t)$.

Assumption 1. There exist constants $\alpha \geq 0$ and $\omega \geq 0$ such that

$$
s(t)=\alpha \sin \omega t
$$

\section{Design and analysis of proposed controller}

In the presence of sensor noise, the proposed output feedback controller equipped with a gain-scaling factor is given by

$$
\begin{aligned}
& \dot{\hat{x}}=A \hat{x}+B v-L(\varepsilon)(\bar{y}-C \hat{x}) \\
& v=K(\varepsilon) \hat{x}-\delta_{4}(\hat{x})
\end{aligned}
$$

where $\hat{x}=\left[\hat{x}_{1}, \hat{x}_{2}, \hat{x}_{3}, \hat{x}_{4}\right]^{T}$ is the observed state, $\delta(\hat{x})=\left[0,0,0, \delta_{4}(\hat{x})\right]^{T}, \delta_{4}(\hat{x})=-\frac{1}{\mu} \hat{x}_{4}, L(\varepsilon)=\left[\frac{l_{1}}{\varepsilon}, \frac{l_{2}}{\varepsilon^{2}}, \frac{l_{3}}{\varepsilon^{3}}, \frac{l_{4}}{\varepsilon^{4}}\right]^{T}$ and $K(\varepsilon)=\left[\frac{k_{1}}{\varepsilon^{4}}, \frac{k_{2}}{\varepsilon^{3}}, \frac{k_{3}}{\varepsilon^{2}}, \frac{k_{4}}{\varepsilon}\right]$ with $\varepsilon>0$. We define $e=\left[e_{1}, e_{2}, e_{3}, e_{4}\right]^{T}$ where $e_{i}=x_{i}-\hat{x}_{i}, i=1, \cdots, 4$. By subtracting (8) from (4) and with the controller (9), the augmented closed-loop dynamics is

$$
\begin{aligned}
& \dot{e}=A_{L}(\varepsilon) e+L(\varepsilon) s(t)+\delta(x)-\delta(\hat{x}) \\
& \dot{x}=A_{K}(\varepsilon) x-B K(\varepsilon) e+\delta(x)-\delta(\hat{x})
\end{aligned}
$$

where $A_{L}(\varepsilon)=A+L(\varepsilon) C$ and $A_{K}(\varepsilon)=A+B K(\varepsilon)$. We must select $L=\left[l_{1}, l_{2}, l_{3}, l_{4}\right]^{T}$ and $K=\left[k_{1}, k_{2}, k_{3}, k_{4}\right]$ such that $A_{L}=A_{L}(1)$ and $A_{K}=A_{K}(1)$ are Hurwitz. The Lyapunov equation for (10) is $A_{L}^{T} P_{L}+P_{L} A_{L}=-I$. With $E(\varepsilon)=\operatorname{diag}\left[1, \varepsilon, \varepsilon^{2}, \varepsilon^{3}\right]$, we have the following equalities [3]:

$$
\begin{aligned}
& \varepsilon A_{L}(\varepsilon)=E(\varepsilon)^{-1} A_{L} E(\varepsilon) \\
& A_{L}{ }^{T}(\varepsilon) P_{L}(\varepsilon)+P_{L}(\varepsilon) A_{L}(\varepsilon)=-\varepsilon^{-1} E(\varepsilon)^{2} \\
& P_{L}(\varepsilon)=E(\varepsilon) P_{L} E(\varepsilon)
\end{aligned}
$$

We set $V_{o}(e)=e^{T} P_{L}(\varepsilon) e$ for $(10)$. Then, along the trajectory of (10), we have

$$
\begin{aligned}
\dot{V}_{o}(e) & =-\varepsilon^{-1} e^{T} E(\varepsilon)^{2} e+2 e^{T} E(\varepsilon) P_{L} E(\varepsilon) L(\varepsilon) s(t)+2 e^{T} E(\varepsilon) P_{L} E(\varepsilon)(\delta(x)-\delta(\hat{x})) \\
& \leq-\varepsilon^{-1}\|E(\varepsilon) e\|^{2}+2 \varepsilon^{-1}\{E(\varepsilon) e\}^{T} P_{L} L s(t)+2\left\|P_{L}\right\|\|E(\varepsilon) e\|\|E(\varepsilon)(\delta(x)-\delta(\hat{x}))\|_{1}
\end{aligned}
$$


We have $\|E(\varepsilon)(\delta(x)-\delta(\hat{x}))\|_{1} \leq \frac{1}{\mu}\|E(\varepsilon) e\|$ for (13), and then we can obtain

$$
\begin{aligned}
\dot{V}_{o}(e) & \leq-\varepsilon^{-1}\|E(\varepsilon) e\|^{2}+2 \varepsilon^{-1}\{E(\varepsilon) e\}^{T} P_{L} L s(t)+2 \frac{2}{\mu}\left\|P_{L}\right\|\|E(\varepsilon) e\|^{2} \\
& \leq-N_{0}\|E(\varepsilon) e\|^{2}-\frac{1}{2} \varepsilon^{-1}\{E(\varepsilon)|e|\}^{T} E(\varepsilon)\left(\left[\begin{array}{l}
\left|e_{1}\right| \\
\left|e_{2}\right| \\
\left|e_{3}\right| \\
\left|e_{4}\right|
\end{array}\right]-4 \alpha\left\|P_{L}\right\|\|L\|\left[\begin{array}{c}
1 \\
\varepsilon^{-1} \\
\varepsilon^{-2} \\
\varepsilon^{-3}
\end{array}\right]\right)
\end{aligned}
$$

where $N_{o}=\frac{1}{2} \varepsilon^{-1}-\frac{2}{\mu}\left\|P_{L}\right\|$ and $|s(t)|=|\alpha \sin \omega t| \leq \alpha$.

Satisfying $N_{o}>0$, the ultimate bound of $e$ is as follows

$$
U B\left(\left[\begin{array}{l}
\left|e_{1}\right| \\
\left|e_{2}\right| \\
\left|e_{3}\right| \\
\left|e_{4}\right|
\end{array}\right]\right) \leq 4 \alpha\left\|P _ { L } \left|\||| L\|\left[\begin{array}{c}
1 \\
\varepsilon^{-1} \\
\varepsilon^{-2} \\
\varepsilon^{-3}
\end{array}\right]\right.\right.
$$

If $\varepsilon$ is increased, the ultimate bounds of $e_{2}, e_{3}$ and $e_{4}$ except for $e_{1}$ can be made arbitrarily small. Because the Lyapunov stability theorem is a sufficient condition, we analyze the ultimate bound of $e_{1}$ via Laplace transform. Applying Laplace transform for the system $(10), E_{1}(s)$ is as follows

$$
E_{1}(s)=\frac{\left(s+\frac{1}{\mu}\right)\left(s^{2} e_{1}(0)+s e_{2}(0)+K_{b b} e_{3}(0)\right)+K_{b b} e_{4}(0)}{\sigma(s)}+\frac{\phi(s)}{\sigma(s)} \frac{\alpha \omega}{s^{2}+\omega^{2}}
$$

where $\sigma(s)=s^{4}+\frac{1}{\mu} s^{3}-\phi(s), \phi(s)=\frac{l_{1}}{\varepsilon} s^{3}+\left(\frac{1}{\mu} \frac{l_{1}}{\varepsilon}+\frac{l_{2}}{\varepsilon^{2}}\right) s^{2}+\left(\frac{1}{\mu} \frac{l_{2}}{\varepsilon^{2}}+K_{b b} \frac{l_{3}}{\varepsilon^{3}}\right) s+\left(\frac{K_{b b}}{\mu} \frac{l_{3}}{\varepsilon^{3}}+K_{b b} \frac{l_{4}}{\varepsilon^{4}}\right)$.

When $\sigma(s)$ is a Hurwitz polynomial, $E_{1}(s)$ applied by the final-value theorem remains as follows

$$
\frac{D_{1}}{s+j \omega}+\frac{D_{2}}{s-j \omega}=\frac{D_{1}(s-j \omega)+D_{2}(s+j \omega)}{s^{2}+\omega^{2}}
$$

where $D_{1}$ and $D_{2}$ is

$$
D_{1}=\frac{\alpha}{2} \frac{\gamma(\omega)+j \beta(\omega)}{\left(\frac{1}{\mu} \omega^{3}-\beta(\omega)\right)-j\left(\omega^{4}-\gamma(\omega)\right)}, D_{2}=\frac{\alpha}{2} \frac{\gamma(\omega)-j \beta(\omega)}{\left(\frac{1}{\mu} \omega^{3}-\beta(\omega)\right)+j\left(\omega^{4}-\gamma(\omega)\right)}
$$

where $\gamma(\omega)=\operatorname{Re}\left(\left.\phi(s)\right|_{s=j w}\right)$ and $\beta(\omega)=\operatorname{Im}\left(\left.\phi(s)\right|_{s=j w}\right)$. Now that $\frac{D_{1}}{s+j \omega}$ and $\frac{D_{2}}{s-j \omega}$ are vibrating terms in steady state, the ultimate bound of $e_{1}$ is minimized if $D_{1}(s-j \omega)+D_{2}(s+j \omega)$, that is the size of (17), is smaller.

$$
\begin{aligned}
& D_{1}(s-j \omega)+D_{2}(s+j \omega) \\
= & \frac{\alpha}{2}\left[\frac{\gamma(\omega)+j \beta(\omega)}{\left(\frac{1}{\mu} \omega^{3}-\beta(\omega)\right)-j\left(\omega^{4}-\gamma(\omega)\right)}(s-j \omega)+\frac{\gamma(\omega)-j \beta(\omega)}{\left(\frac{1}{\mu} \omega^{3}-\beta(\omega)\right)+j\left(\omega^{4}-\gamma(\omega)\right)}(s+j \omega)\right] \\
= & \alpha\left[\frac{\varepsilon^{8} \frac{1}{\mu} \gamma(\omega) \omega^{3}-\varepsilon^{8} \beta(\omega) \omega^{4}}{\varepsilon^{8}\left(\frac{1}{\mu} \omega^{3}-\beta(\omega)\right)^{2}+\varepsilon^{8}\left(\omega^{4}-\gamma(\omega)\right)^{2}} s+\frac{\varepsilon^{8} \gamma(\omega) \omega^{4}+\varepsilon^{8} \frac{1}{\mu} \beta(\omega) \omega^{3}-\varepsilon^{8} \gamma(\omega)^{2}-\varepsilon^{8} \beta(\omega)^{2}}{\varepsilon^{8}\left(\frac{1}{\mu} \omega^{3}-\beta(\omega)\right)^{2}+\varepsilon^{8}\left(\omega^{4}-\gamma(\omega)\right)^{2}}\right]
\end{aligned}
$$


Substituting $\gamma(\omega)=\operatorname{Re}\left(\left.\phi(s)\right|_{s=j w}\right)$ and $\beta(\omega)=\operatorname{Im}\left(\left.\phi(s)\right|_{s=j w}\right)$ into (18), we can rewrite (18) for $\varepsilon$ as follows

$$
D_{1}(s-j \omega)+D_{2}(s+j \omega)=\alpha\left[\frac{l_{1}\left(\omega^{7}-\frac{1}{\mu} \omega^{5}\right) \varepsilon^{7}+O\left(\varepsilon^{6}\right)}{\left(\omega^{8}+\frac{1}{\mu} \omega^{6}\right) \varepsilon^{8}+O\left(\varepsilon^{7}\right)} s+\frac{\left(l_{1}^{2} \omega^{6}-l_{2} \omega^{6}+\frac{1}{\mu^{2}} l_{1}^{2} \omega^{4}\right) \varepsilon^{6}+O\left(\varepsilon^{5}\right)}{\left(\omega^{8}+\frac{1}{\mu} \omega^{6}\right) \varepsilon^{8}+O\left(\varepsilon^{7}\right)} \omega\right]
$$

If $\varepsilon$ is increased, the size of $D_{1}(s-j \omega)+D_{2}(s+j \omega)$ is decreased. Thus, the ultimate bound of $e_{1}$ can be reduced by increasing $\varepsilon$. The Lyapunov equation for (11) is $A_{K}^{T} P_{K}+P_{K} A_{K}=-I$, and we have the following equalities:

$$
\begin{aligned}
& \varepsilon A_{K}(\varepsilon)=E(\varepsilon)^{-1} A_{K} E(\varepsilon) \\
& A_{K}{ }^{T}(\varepsilon) P_{K}(\varepsilon)+P_{K}(\varepsilon) A_{K}(\varepsilon)=-\varepsilon^{-1} E(\varepsilon)^{2} \\
& P_{K}(\varepsilon)=E(\varepsilon) P_{K} E(\varepsilon)
\end{aligned}
$$

We set $V_{c}(x)=x^{T} P_{K}(\varepsilon) x$ for (11). Then, along the trajectory of (11), we have

$$
\begin{aligned}
\dot{V}_{c}(x) & =-\varepsilon^{-1}\|E(\varepsilon) x\|^{2}-2 \varepsilon^{-1} x^{T} E(\varepsilon) P_{K} B K E(\varepsilon) e+2 x^{T} E(\varepsilon) P_{K} E(\varepsilon)\{\delta(x)-\delta(\hat{x})\} \\
& \leq-\varepsilon^{-1}\|E(\varepsilon) x\|^{2}-2 \varepsilon^{-1}\{E(\varepsilon) x\}^{T} P_{K} B K E(\varepsilon) e+2 q\|E(\varepsilon) x\|\|E(\varepsilon) e\|
\end{aligned}
$$

where $q=\frac{1}{\mu}\left\|P_{K}\right\|$.

We set a Lyapunov function for entire system (10)-(11) to $V_{c}(e, x)=V_{o}(e)+d V_{c}(x), d>0$. Then, along the trajectories of (10) and (11), we obtain the following from (14) and (21).

$$
\begin{aligned}
\dot{V}(e, x) \leq & -\sum^{T} M \sum-\frac{1}{2} \varepsilon^{-1}\{E(\varepsilon)|e|\}^{T} E(\varepsilon)\left(\left[\begin{array}{l}
\left|e_{1}\right| \\
\left|e_{2}\right| \\
\left|e_{3}\right| \\
\left|e_{4}\right|
\end{array}\right]-4 \alpha\left\|P_{L}\right\|\|L\|\left[\begin{array}{c}
1 \\
\varepsilon^{-1} \\
\varepsilon^{-2} \\
\varepsilon^{-3}
\end{array}\right]\right) \\
& -\frac{1}{2} \varepsilon^{-1} d\{E(\varepsilon)|x|\}^{T} E(\varepsilon)\left(\left[\begin{array}{l}
\left|x_{1}\right| \\
\left|x_{2}\right| \\
\left|x_{3}\right| \\
\left|x_{4}\right|
\end{array}\right]-4\left\|P_{K}\right\|\|K\|\left[\begin{array}{c}
1 \\
\varepsilon^{-1} \\
\varepsilon^{-2} \\
\varepsilon^{-3}
\end{array}\right]\left(\left|e_{1}\right|+\varepsilon\left|e_{2}\right|+\varepsilon^{2}\left|e_{3}\right|+\varepsilon^{3}\left|e_{4}\right|\right)\right)
\end{aligned}
$$

where

$$
\sum=\left[\begin{array}{l}
\|E(\varepsilon) e\| \\
\|E(\varepsilon) x\|
\end{array}\right], M=\left[\begin{array}{cc}
N_{o} & -d q \\
-d q & \frac{d}{2} \varepsilon^{-1}
\end{array}\right]
$$

If $d<\frac{N_{o}}{2 \varepsilon q^{2}}$ is satisfied, $M$ becomes positive definite matrix. Thus, the ultimate bound of $\left(x_{1}, x_{2}, x_{3}, x_{4}\right)$ is as follows

$$
U B\left(\left[\begin{array}{c}
\left|x_{1}\right| \\
\left|x_{2}\right| \\
\left|x_{3}\right| \\
\left|x_{4}\right|
\end{array}\right]\right) \leq 4\left\|P_{K}\right\|\|K\|\left[\begin{array}{c}
1 \\
\varepsilon^{-1} \\
\varepsilon^{-2} \\
\varepsilon^{-3}
\end{array}\right]\left(\left|e_{1}\right|+\varepsilon\left|e_{2}\right|+\varepsilon^{2}\left|e_{3}\right|+\varepsilon^{3}\left|e_{4}\right|\right)
$$


Substituting the ultimate bound of $\left(e_{1}, e_{2}, e_{3}, e_{4}\right)$ into (23), we can obtain

$$
U B\left(\left[\begin{array}{l}
\left|x_{1}\right| \\
\left|x_{2}\right| \\
\left|x_{3}\right| \\
\left|x_{4}\right|
\end{array}\right]\right) \leq 64 \alpha\left\|P_{K}\right\|\|K\|\left\|P_{L}\right\|\|L\|\left[\begin{array}{c}
1 \\
\varepsilon^{-1} \\
\varepsilon^{-2} \\
\varepsilon^{-3}
\end{array}\right]
$$

If $\varepsilon$ is increased in (24), the ultimate bounds of $x_{2}, x_{3}$ and $x_{4}$ is decreased except for $x_{1}$. Thus, we analyze the ultimate bound of $x_{1}$ via Laplace transform.

Applying Laplace transform for the system (11), $X_{1}(s)$ is as follows

$$
\begin{aligned}
X_{1}(s)= & \frac{s^{3}-\left(\frac{k_{4}}{\varepsilon}-\frac{1}{\mu}\right) s^{2}-\frac{k_{3}}{\varepsilon^{2}} s-\frac{K_{b b} k_{2}}{\varepsilon^{3}}}{\eta(s)} x_{1}(0)+\frac{s^{2}-\left(\frac{k_{4}}{\varepsilon}-\frac{1}{\mu}\right) s-\frac{k_{3}}{\varepsilon^{2}}}{\eta(s)} x_{2}(0)+\frac{\left(s-\frac{k_{4}}{\varepsilon}+\frac{1}{\mu}\right) K_{b b}}{\eta(s)} x_{3}(0) \\
& +\frac{K_{b b}}{\eta(s)} x_{4}(0)+\frac{1}{\eta(s)} \frac{K_{s r v}}{\mu}\left(\frac{k_{1}}{\varepsilon^{4}} E_{1}(s)+\frac{k_{2}}{\varepsilon^{3}} E_{2}(s)+\frac{k_{3}}{\varepsilon^{2}} E_{3}(s)+\frac{k_{4}}{\varepsilon} E_{4}(s)\right)
\end{aligned}
$$

where $\eta(s)=s^{4}-\left(\frac{k_{4}}{\varepsilon}-\frac{1}{\mu}\right) s^{3}-\frac{k_{3}}{\varepsilon^{2}} s^{2}-\frac{k_{2} K_{b b}}{\varepsilon^{3}} s-\frac{k_{1} K_{b b}}{\varepsilon^{4}}$.

When $\eta(s)$ is a Hurwitz polynomial, the terms with initial value for (25) become 0 by the final-value theorem and the remaining terms $\left(X_{r 1}(s)\right)$ is as follows

$$
X_{r 1}(s)=\frac{1}{\eta(s)} \frac{K_{s r v}}{\mu}\left(\frac{k_{1}}{\varepsilon^{4}} E_{1}(s)+\frac{k_{2}}{\varepsilon^{3}} E_{2}(s)+\frac{k_{3}}{\varepsilon^{2}} E_{3}(s)+\frac{k_{4}}{\varepsilon} E_{4}(s)\right)
$$

We assume that $\eta(s)$ has separate roots for analysis and substitute $E_{b i}(s), i=1, \cdots, 4$, that is the ultimate bound of $E_{i}(s), i=1, \cdots, 4$, into (26) as follows.

$$
\frac{\frac{K_{s v}}{\mu}}{\left(s-\lambda_{1}\right)\left(s-\lambda_{2}\right)\left(s-\lambda_{3}\right)\left(s-\lambda_{4}\right)}\left(\frac{k_{1}}{\varepsilon^{4}} E_{b 1}(s)+\frac{k_{2}}{\varepsilon^{3}} E_{b 2}(s)+\frac{k_{3}}{\varepsilon^{2}} E_{b 3}(s)+\frac{k_{4}}{\varepsilon} E_{b 4}(s)\right)=\left(\sum_{i=1}^{4} \frac{a_{i}}{s+\lambda_{i}}\right)\left(\sum_{i=1}^{4} \frac{k_{i}}{\varepsilon^{5-i}} E_{b i}(s)\right)(27
$$

where $\lambda_{i}, i=1, \cdots, 4$ are roots of $\eta(s), a_{i}, i=1, \cdots, 4$ are constants by partial fraction expansion for $\frac{K_{s v} / \mu}{\left(s-\lambda_{1}\right)\left(s-\lambda_{2}\right)\left(s-\lambda_{3}\right)\left(s-\lambda_{4}\right)}$. Applying the inversion of Laplace transform for (27)

$$
\left(\sum_{i=1}^{4} a_{i} \exp \left(-\lambda_{i} t\right)\right) *\left(\sum_{i=1}^{4} \frac{k_{i}}{\varepsilon^{5-i}} e_{b i}(t)\right)=\int_{0}^{t}\left(\sum_{i=1}^{4} a_{i} \exp \left(-\lambda_{i}(t-\tau)\right)\right) *\left(\sum_{i=1}^{4} \frac{k_{i}}{\varepsilon^{5-i}} e_{b i}(\tau)\right) d \tau
$$

Because the ultimate bounds of $\left(x_{1}, x_{2}, x_{3}, x_{4}\right)$ are reduced by increasing $\varepsilon$ as shown in (15) and (19), we set maximum value of $e_{b i}(t)$ as $\frac{b_{i}}{\varepsilon}, i=1, \cdots, 4$.

$$
\int_{0}^{t}\left(\sum_{i=1}^{4} a_{i} \exp \left(-\lambda_{i}(t-\tau)\right)\right) *\left(\sum_{i=1}^{4} \frac{k_{i}}{\varepsilon^{5-i}} e_{b i}(\tau)\right) d \tau \leq\left(\sum_{i=1}^{4} \frac{a_{i}}{\lambda_{i}}\left[1-\exp \left(-\lambda_{i} t\right)\right]\right)\left(\sum_{i=1}^{4} \frac{k_{i}}{\varepsilon^{5-i}} \frac{b_{i}}{\varepsilon}\right)
$$

The exponential terms $\exp \left(-\lambda_{i} t\right), i=1, \cdots, 4$ become 0 in steady state. Thus, the ultimate bound of $x_{1}$ in steady state is as follows

$$
U B\left(x_{1}\right) \leq \frac{1}{\varepsilon}\left(\frac{a_{1}}{\lambda_{1}}+\frac{a_{2}}{\lambda_{2}}+\frac{a_{3}}{\lambda_{3}}+\frac{a_{4}}{\lambda_{4}}\right)\left(\frac{k_{1} b_{1}}{\varepsilon^{4}}+\frac{k_{2} b_{2}}{\varepsilon^{3}}+\frac{k_{3} b_{3}}{\varepsilon^{2}}+\frac{k_{4} b_{4}}{\varepsilon}\right)
$$

If $\varepsilon$ is increased in (30), the ultimate bounds of $x_{1}, x_{2}, x_{3}$ and $x_{4}$ can be reduced. 


\section{Simulation}

In simulation for a ball and beam system, we set the initial conditions as $x_{1}(0)=10 \mathrm{~cm}, x_{2}(0)=0$, $x_{3}(0)=0^{\circ}$ and $x_{4}(0)=0$ and sensor noise as $s(t)=3 \sin 3 t$. The matrices $A_{L}$ and $A_{K}$ are Hurwitz if choosing $L=[-100,-3080,-71235,1567]^{T}$ and $K=[-0.3490,-0.8725,-0.3242,0.5320]$. When the value of $\varepsilon$ changed, the position of ball and the angle of motor are shown as Fig. 1 and Fig. 2. As shown in Fig. 1 and Fig. 2, the ultimate bounds of $x_{1}$ and $x_{3}$ is reduced when $\varepsilon$ is increased. Thus, our proposed controller can reduce the effect of sensor noise by increasing $\varepsilon$.

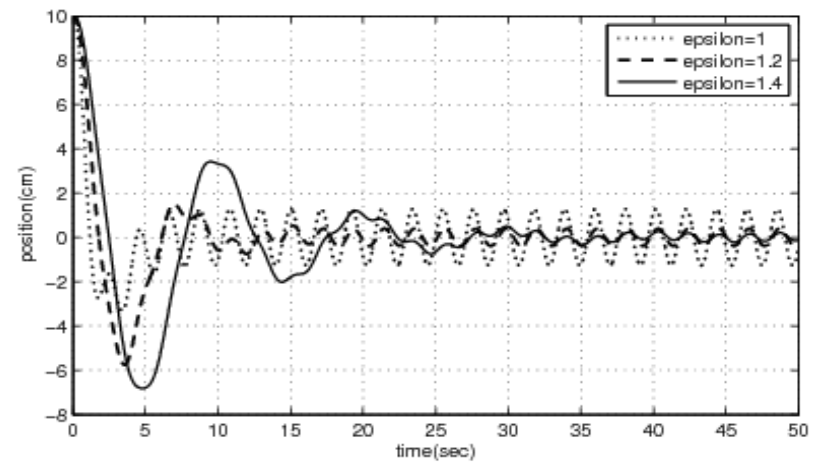

Fig. 1. Position of ball $\left(x_{1}\right)$

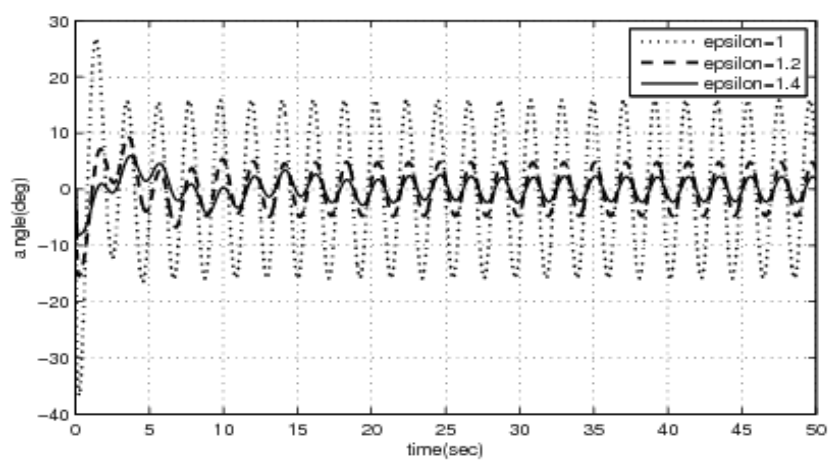

Fig. 2. Angle of motor $\left(x_{3}\right)$

\section{Conclusions}

In this paper, we assume that AC sensor noise is included in the output sensor of a ball and beam system. We design an output feedback controller with a gain-scaling factor to minimize the effect of sensor noise. We analyze the ultimate bounds of $e$ and $x$ by the Lyapunov stability theorem and Laplace transform for controlled system. As a result, we show that the effect of sensor noise is arbitrarily reduced by increasing $\varepsilon$ via analysis and simulation.

\section{Acknowledgement}

This research was supported by Basic Science Research Program through the National Research Foundation of Korea (NRF) funded by the Ministry of Education, Science and Technology (2010-0007325).

\section{References}

[1] C.-T. Chen, Linear system theory and design, 3rd Ed., Oxford University Press Inc., 1999.

[2] Z. Chen, A remark on sensor disturbance rejection of nonlinear systems, IEEE Trans. Autom. Control, vol. 54, no. 9, pp. 2206-2210, 2009.

[3] H.-L. Choi and J.-T. Lim, Output feedback stabilization for a class of Lipschitz nonlinear systems, IEICE Trans. Fundamentals, vol. E88-A, no. 2, pp. 602-605, 2005.

[4] H. K. Khalil, Nonlinear systems, 3rd, Prentice Hall Inc., 2002.

[5] H.-D. Kim and H.-L. Choi, Design and analysis of an output feedback controller for a chain of integrators system compensating measurement noise of feedback sensor, Journal of Institute of Control, Robotics and Systems (in Korean), vol. 17, no. 4, pp. 299-303, 2011.

[6] T. B. Sekara and M. R. Matausek, Optimization of PID controller based on maximization of the proportional gain under constraints on robustness and sensitivity to measurement noise, IEEE Trans. Autom. Control, vol. 54, no 1, pp. 184-189, 2009.

[7] Quanser, Ball and beam user manuals, 2008. 